\title{
Agglomeration of wet particles in dense granular flows
}

\author{
Thanh Trung Vo ${ }^{1,2}$, Saeid Nezamabadi ${ }^{1,3, a}$, Patrick Mutabaruka ${ }^{1}$, Jean-Yves Delenne ${ }^{3}$, Edouard Izard ${ }^{4}$, \\ Roland Pellenq ${ }^{5}$, and Farhang Radjai ${ }^{1}$ \\ 1 LMGC, Université de Montpellier, CNRS, Montpellier, France \\ 2 Bridge and Road Department, Danang Architecture University, 553000 Da Nang, Vietnam \\ 3 IATE, UMR1208 INRA - CIRAD - Université de Montpellier - SupAgro, 34060 Montpellier, France \\ 4 ArcelorMittal R\&D Maizières, Voie Romaine, F-57283 Maizières-Lès-Metz, France \\ 5 (MSE2), UMI 3466 CNRS-MIT, MIT Energy Initiative, \\ 77 Massachusetts Avenue, Cambridge, MA 02139, USA
}

\begin{abstract}
In order to get insight into the wet agglomeration process, we numerically investigate the growth of a single granule inside a dense flow of an initially homogeneous distribution of wet and dry particles. The simulations are performed by means of the discrete element method and the binding liquid is assumed to be transported by the wet particles, which interact via capillary and viscous force laws. The granule size is found to be an exponential function of time, reflecting the conservation of the amount of liquid and the decrease of the number of available wet particles inside the flow during agglomeration. We analyze this behavior in terms of the accretion and erosion rates of wet particles for a range of different values of material parameters such as mean particle size, size polydispersity, friction coefficient and liquid viscosity. In particular, we propose a phase diagram of the granule growth as a function of the mean primary particle diameter and particle size span, which separates the parametric domain in which the granule grows from the domain in which the granule does not survive.
\end{abstract}

\section{Introduction}

Wet granulation or agglomeration of fine solid particles into larger particles, called granules or agglomerates, is a widespread technique in industrial processes such as the manufacture of pharmaceuticals $[1,2]$, fertilizers and food products $[3,4]$, powder metallurgy [5] and iron-making [68]. The increased size of the granules modifies the rheological properties of the granular material and may improve flow properties, reduce the segregation of different types of primary particles or enhance the permeability for the interstitial gas between grains [4,9-13]. The wetting of primary particles is achieved either by mixing them with a binding liquid prior to the process or by dripping or spraying the liquid to the material during the process [13-21]. The granules nucleate as a result of the collisional-frictional/capillary-viscous interactions of the wet primary particles and then they grow in size by incorporation of the available liquid, accretion of primary finer particles and coalescence with other granules [22]. The existing granules may survive and grow or disappear, depending on the amount of available liquid and the rate

\footnotetext{
a e-mail: saeid.nezamabadi@umontpellier.fr
}

of erosion as compared to that of accretion (layering) and coalescence $[1,23]$.

Given the large number of parameters involved in the agglomeration process, its detailed physical mechanisms and their relative importance for the resulting properties of are complex. One may distinguish two different groups of parameters related to the material and the process $[9$, 10,24-27]. The material parameters are the properties of the binding liquid and raw material such as liquid viscosity, primary particle size distribution, mean particle size and friction coefficient of primary particles $[19,28,29]$. The process parameters are related to the method of mixing solid particles with the binding liquid and the corresponding operating parameters. There are different types of granulators using fluidized beds, high shear or low shear in planetary devices or rotating drums or disks [9]. Major process parameters in all types of granulators are the liquid volume $[8,25,28]$, granulator size, rotation speed, inclination angle [30-32] and filling rate [33]. The agglomeration process often needs to be optimized by playing with all these parameters in order to produce granules of high density, homogeneous distribution of primary particles, a targeted mean size and high strength [34-38]. 
The agglomeration process is easier to model and control when particle dynamics is governed by binary collisions, as in granulators based on fluidized bed or high shearing by impellers. Such processes have been extensively investigated in application to the pharmaceutical industry [10]. In contrast, in drum granulators the particles agglomerate in a downward dense granular flow along inclined rotating drum. The drum granulator has the advantage of being a continuous and robust process, but since the rheology of dense granular flows is a matter of current research [39-41], the agglomeration mechanisms in this geometry remain quite poorly understood $[9,42]$. Granular flows in an inclined rotating drum may show several flow regimes [43-45] with the common feature of being dense and inhomogeneous, and involving inertial effects $[14,18$, 23,46-49]. A practical difficulty with drum agglomeration is the in-line monitoring of the kinetics, making it less amenable to theoretical understanding, which is required in order to be able to improve drum granulation plants, often suffering from a significant recycle of undersize and crushed oversize granules [9].

In this paper, we use the drum geometry to create a steady dense flow of spherical particles in order to investigate the growth of a single pre-nucleated granule composed of wet particles acting as a seed. The granule can grow by capturing free wet particles inside the granular flow, or shrink by losing its wet particles. The particle dynamics is simulated by the Discrete Element Method (DEM) [50], but the binding liquid is accounted for via inter-particle capillary and viscous forces. In other words, it is assumed that the wet particles have a large moisture content either in the form of a liquid layer at the surface of the particles $[1,22]$ or in the volume of small aggregates of finer particles glued together by the liquid that they transport. Upon contact, such wet particles form a binary liquid bridge whose shape is governed by the Laplace-Young equation. The capillary force law used in this work is an explicit approximative solution of this equation. We also account for the debonding distance as a function of the amount of liquid in a capillary bridge [51].

The DEM allows for direct quantification of particlescale kinetics and accretion/erosion events. This method has already been applied to investigate agglomeration in granular shear flows. For example, Talu et al. [52] introduced a model of wet granulation in which some of the particles are assumed to be covered by a binder and therefore sticky while the rest are non-cohesive. The binder layer between particles dissipates energy due to viscosity and allows the particles to stick to one another by the action of capillary forces. In other reported DEM simulations of the granulation process, besides capillary and viscous forces, simple empirical rules are used for progressive wetting of the particles [53-55]. To reduce the high computational cost of DEM simulations, some authors have used the DEM simulation data with a low number of particles to train an artificial neural network or in conjunction with population balance equations for application to the large number of particles $[54,56]$. The DEM has, however, only recently been employed for drum granulation using a JKR elastic-adhesive force law with a scale-up procedure based on the cohesion number [42]. In the work presented in this paper, we consider idealized particles, and drum rotation is used only as a means to sustain a dense continuous flow while we investigate the influence of mean particles size, size ratio, liquid viscosity and friction coefficient on the granule growth.

In the following, in sect. 2, we introduce in detail the numerical approach. Then, we analyze in sect. 3 the growth process in terms of accretion and erosion events, and in sect. 4 we investigate the influence of material parameters on the accretion and erosion rates. In sect. 5 , we introduce a phase diagram for granule growth as a function of the mean particle size and size ratio. Finally, we conclude in sect. 6 with a short summary and future research directions.

\section{Model description and numerical method}

Our numerical approach for the simulation of the agglomeration process is based on the DEM and a model for the effect of the binding liquid. We first describe below the physical assumptions underlying the model. Then, we briefly present the numerical algorithm with its input parameters and main calculation steps.

\subsection{Physical assumptions}

In the DEM, the equations of motion of all particles are integrated according to an explicit time-stepping scheme such as the well-known velocity-Verlet algorithm [50,5761]. A detailed description of the liquid phase and its interaction with solid particles, requires sub-particle discretization of the liquid phase and a numerical model for liquidgas phase transition $[41,47,49]$. However, such a multicomponent model of partially saturated granular materials is not computationally efficient for the simulation of the agglomeration process with a large number of primary particles. An efficient alternative approach consists in accounting for the capillary and lubrication forces between particles as well as a particle-scale model for the distribution and transport of the binding liquid.

Recent experiments and numerical simulations show that the liquid clusters condensed from a vapor or introduced by mixing the liquid with particles can be characterized by their connectivity with the particles $[47,49]$. The number of liquid clusters connected to two particles prevails for low amounts of the liquid. In this "pendular" state, the liquid is in the form of binary bridges. As the amount of liquid increases, the clusters involve more and more particles until a single cluster spans the whole packing. The cohesive effect of the liquid in thermodynamic equilibrium is controlled by the total wetted surface and the Laplace pressure. The cohesion rapidly increases as the amount of liquid is increased in the pendular state, and then it keeps a nearly constant value (or slightly increases) with increasing amount of the liquid before declining for large amounts $[47,49]$. This description assumes, however, that the particles are in quasi-static equilibrium 
and the liquid is in thermodynamic equilibrium. The negative Laplace pressure within the liquid phase is not uniformly distributed if the system is out of equilibrium. Furthermore, if the granular material flows, the liquid clusters undergo large distortions, and the liquid is continuously re-distributed as a result of coalescence and separation of liquid clusters $[62,63]$. In practice, a small amount of the added liquid is adsorbed into the particle rough surfaces and is not directly involved in capillary bonding between particles.

The above-mentioned features suggest that in a dense granular material, for a broad range of the amount of liquid, the cohesive capillary stress is nearly independent of the amount of liquid, and therefore the effect of liquid volume can be accounted for by the number of wet particles. In a rotating drum, when the liquid is poured onto the granular flow, it has not time to diffuse and the capillary stress leads to the creation of small aggregates of primary particles that are transported by the granular flow. These "micro-aggregates" may deform or break up into smaller aggregates [1]. They may also capture more primary particles or coalesce into larger aggregates if they have an excess amount of liquid that can be shared with other microaggregates as a consequence of their consolidation under the action of contact forces inside the granular flow. The initial size of the micro-aggregates is proportional to that of the droplets but, depending on the wetting method, it can grow rapidly into granules.

This picture of liquid transport by micro-aggregates in their pore volumes or at their surfaces in the form of liquid layer covering the asperities means that the discreteelement model can be based on the micro-aggregates as wet units. In its simplest setting, these basic units can be modeled as particles each transporting a given amount of liquid. Since the number of primary particles embodied in each micro-aggregate is nearly proportional to the droplet size, the size distribution of the basic particles can be regarded as reflecting that of the droplets. When two such wet particles meet, they are subjected to the cohesive action of the spontaneous liquid bridge appearing between them. In this sense, the interactions between wet units are similar to those between particles covered by a liquid layer as in the method introduced by Talu et al. [52] or indirectly through the use of surface tension as in [42]. In the following, we present in more detail our numerical method and all model parameters.

As in this model the liquid is mainly transported by the population of wet particles, the initial distribution and amount of the binding liquid are essentially determined by those of wet particles. It is, however, important to note that we do not consider here the nucleation step in which the processes of wetting and dispersion of the binding liquid are crucial for the early-stage evolution of the system and for which a fluid-resolved method is necessary $[1,49,64,65]$. Once the granules are nucleated, the subsequent granule growth is mainly controlled by the accretion/erosion mechanisms, and liquid re-distribution takes place by the interactions between the granules and wet or dry particles. In our simulations, we may control the initial state by changing the initial distribution of free wet particles in order to analyze its influence on the growth kinetics of the granules. We will consider below the simple situation of a random distribution of wet particles. Hence, the growth process is mainly governed by the accretion of these free wet particles by a pre-nucleated granule and the erosion of particles from granule by interaction with dry particles without fluctuations arising from an uneven distribution of the binding liquid. Several previous studies of agglomeration suggest that this approximation provides results that are in good agreement with experiments when the material parameters are correctly adjusted $[42,52]$.

\subsection{Numerical method}

In the DEM, the particles interact via viscoelastic forces as a function of the local contact strain defined from the relative displacements of the particles. Since the particles are assumed to be rigid, a large repulsive stiffness and hence a high time resolution are required for the computation of the interactions between particles. The motion of each rigid spherical particle $i$ of radius $R_{i}$ is governed by Newton's second law under the action of normal contact forces $f_{n}$, tangential contact forces $f_{t}$, capillary forces $f_{c}$, viscous forces $f_{v i s}$ and particle weight $m_{i} \mathbf{g}$

$$
\begin{aligned}
m_{i} \frac{\mathrm{d}^{2} \mathbf{r}_{i}}{\mathrm{~d} t^{2}} & =\sum_{j}\left[\left(f_{n}^{i j}+f_{c}^{i j}+f_{v i s}^{i j}\right) \mathbf{n}^{i j}+f_{t}^{i j} \mathbf{t}^{i j}\right]+m_{i} \mathbf{g}, \\
\mathbf{I}_{i} \frac{\mathrm{d} \boldsymbol{\omega}_{i}}{\mathrm{~d} t} & =\sum_{j} f_{t}^{i j} \mathbf{c}^{i j} \times \mathbf{t}^{i j},
\end{aligned}
$$

where $\boldsymbol{\omega}_{i}$ is the rotation vector of particle $i$, and $m_{i}, \mathbf{I}_{i}, \mathbf{r}_{i}$ and $\mathbf{g}$ are the mass, inertia matrix, position and gravity acceleration vector of particle $i$, respectively. $\mathbf{n}^{i j}$ denotes the unit vector perpendicular to the contact plane with particle $j$ and pointing from $j$ to $i, \mathbf{t}^{i j}$ is the unit vector belonging to the contact plane $i j$ and pointing in the direction opposite to the relative displacement of the two particles and $\mathbf{c}^{i j}$ is the vector pointing from the center of particle $i$ to the contact point with particle $j$. The tangential viscous dissipation, as compared to the normal lubrication force, is neglected [66]. The equations of motion are integrated according to a velocity-Verlet time-stepping scheme $[50,67]$.

The normal contact force $f_{n}$ involves two components $[68,69]$,

$$
f_{n}=f_{n}^{e}+f_{n}^{d}
$$

The normal elastic force $f_{n}^{e}=k_{n} \delta_{n}$ is a linear function of the normal elastic deflection $\delta_{n}$, where $k_{n}$ is the normal stiffness constant, and the normal damping force $f_{n}^{d}=\gamma_{n} \dot{\delta}_{n}$ is proportional to the relative normal velocity $\dot{\delta}_{n}$, where $\gamma_{n}$ is the normal viscous damping parameter. These both forces appear only when there is overlap, i.e. for $\delta_{n}<0$.

The tangential contact force $f_{t}$ is the sum of an elastic force $f_{t}^{e}=k_{t} \delta_{t}$ and a damping force $f_{t}^{d}=\gamma_{t} \dot{\delta}_{t}$, where $k_{t}$ is the tangential stiffness, $\gamma_{t}$ denotes the tangential viscous 


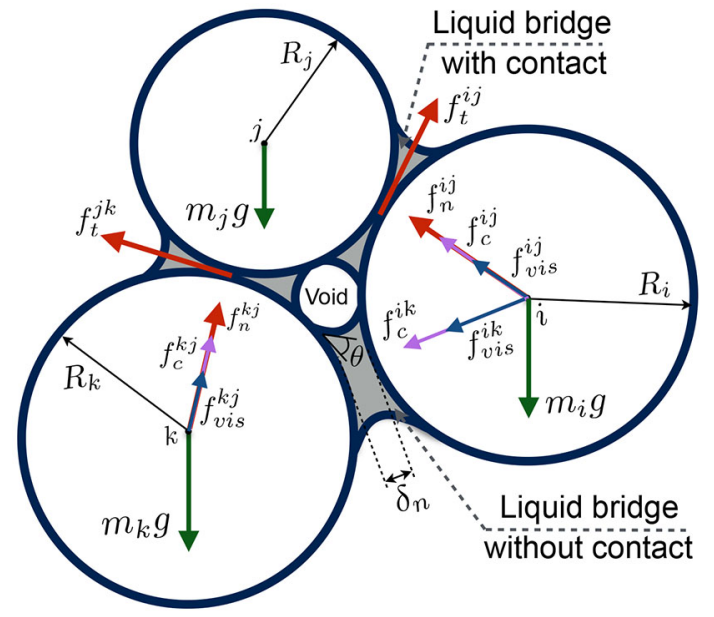

Fig. 1. Schematic drawing of two different cases of capillary bridges: particle $i$ in contact with particle $j$ and without contact with particle $k$.

damping parameter and $\delta_{t}$ and $\dot{\delta}_{t}$ are the tangential displacement and velocity in contact, respectively. According to the Coulomb friction law, the tangential force is bounded by a force threshold $\mu f_{n}$, where $\mu$ is the friction coefficient $[51,70-72]$

$$
f_{t}=-\min \left\{\left(k_{t} \delta_{t}+\gamma_{t} \dot{\delta}_{t}\right), \mu f_{n}\right\}
$$

The capillary attraction force $f_{c}$ between two particles depends on the liquid volume $V_{b}$ of the bond, liquidvapor surface tension $\gamma_{s}$ and particle-liquid-gas contact angle $\theta$; see fig. $1[46,68,73]$. The capillary force is given by the Laplace-Young equation. In the pendular state, an approximate solution is given by the expression $[51,74]$

$$
f_{c}= \begin{cases}-\kappa R, & \text { for } \delta_{n}<0 \\ -\kappa R e^{-\delta_{n} / \lambda}, & \text { for } 0 \leq \delta_{n} \leq d_{\text {rupt }} \\ 0, & \text { for } \delta_{n}>d_{\text {rupt }}\end{cases}
$$

where $R=\sqrt{R_{i} R_{j}}$ is the geometrical mean radius of two particles of radii $R_{i}$ and $R_{j}$ and the capillary force prefactor $\kappa$ is [51]

$$
\kappa=2 \pi \gamma_{s} \cos \theta
$$

This force exists up to a debonding distance $d_{\text {rupt }}$ defined by [46]

$$
d_{\text {rupt }}=\left(1+\frac{\theta}{2}\right) V_{b}^{1 / 3} .
$$

The characteristic length $\lambda$ in eq. (4) is given by

$$
\lambda=\operatorname{ch}(r)\left(\frac{V_{b}}{R^{\prime}}\right)^{1 / 2},
$$

where $R^{\prime}=2 R_{i} R_{j} /\left(R_{i}+R_{j}\right)$ and $r=\max \left\{R_{i} / R_{j} ; R_{j} / R_{i}\right\}$ are the harmonic mean radius and the size ratio between two particles. Figure 2(a) displays the capillary force as a function of the gap between two particles for different

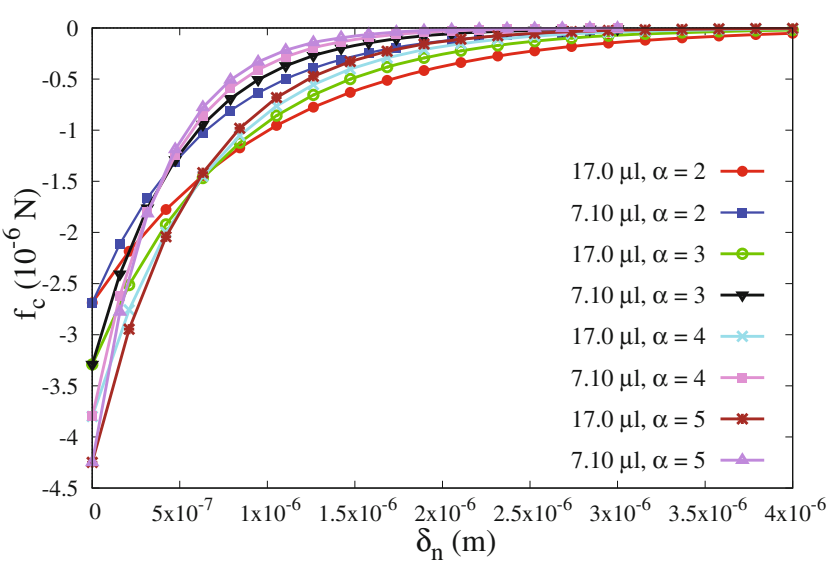

(a)

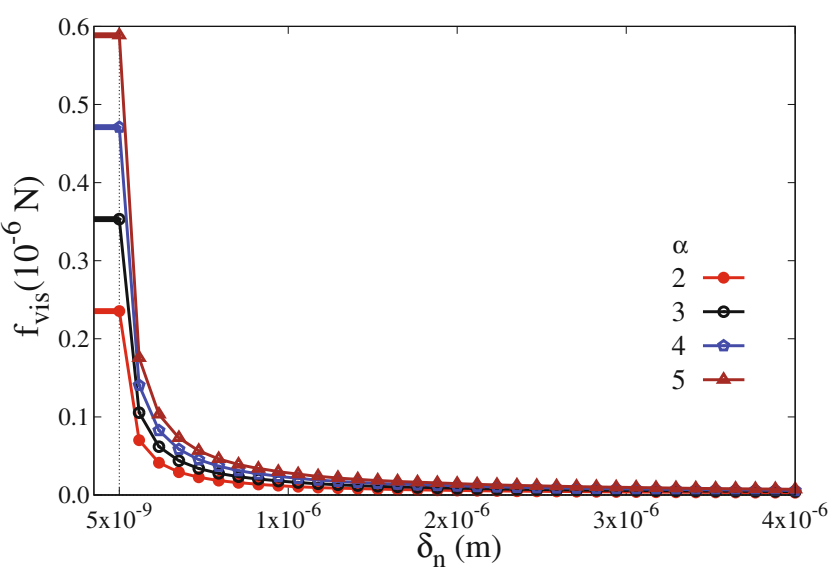

(b)

Fig. 2. (a) Capillary cohesion force $f_{c}$ as a function of the gap $\delta_{n}$ between two particles for different values of the liquid volume $V_{b}(\mu \mathrm{l})$ and size ratio $\alpha$; (b) Lubrication force as a function of the gap between two particles for given values of relative velocity $\left(5 \times 10^{-3} \mathrm{~m} / \mathrm{s}\right)$ and liquid viscosity $(\eta=1 \mathrm{mPa} \cdot \mathrm{s})$ and different values of size ratio $\alpha$.

values of size ratio $\alpha$ and liquid volume $V_{b}$. The expression (4) nicely fits the capillary force as obtained from direct integration of the Laplace-Young equation by setting $h(r)=r^{-1 / 2}$ and $c \simeq 0.9[51,68,75]$. It is also very close to the expression used in [76].

The normal viscous force $f_{v i s}$ is due to the lubrication effect of liquid bridges between particles. Its classical expression for two smooth spherical particles is $[66,77]$

$$
f_{v i s}=\frac{3}{2} \pi R^{2} \eta \frac{v_{n}}{\delta_{n}}
$$

where $\eta$ is the liquid viscosity and $v_{n}$ is the relative normal velocity assumed to be positive when the gap $\delta_{n}$ is decreasing. This expression implies that the viscous force diverges when the gap $\delta_{n}$ tends to zero. With this singularity, two rigid particles cannot collide in finite time. However, for slightly rough particles, the surfaces are no longer parallel and the characteristic size of the asperities 
allows for collision in finite time. Hence, we introduce a characteristic length $\delta_{n 0}$ representing the size of asperities and assume that the lubrication force is given by

$$
f_{v i s}=\frac{3}{2} \pi R^{2} \eta \frac{v_{n}}{\delta_{n}+\delta_{n 0}} \quad \text { for } \delta_{n}>0
$$

as long as $\delta_{n}>0$, i.e. for a positive gap. This expression ensures that the singularity will not occur as long as there is no contact. When a contact occurs, i.e. for $\delta_{n}<0$, we assume that the lubrication force remains equal to its largest value given by

$$
f_{v i s}=\frac{3}{2} \pi R^{2} \eta \frac{v_{n}}{\delta_{n 0}} \quad \text { for } \delta_{n} \leq 0
$$

Figure 2(b) shows $f_{v i s}$ as a function of $\delta_{n}$ between two particles for a given value of the relative normal velocity and liquid viscosity. In our simulations, we set $\delta_{n 0}=5$. $10^{-4} d_{\text {min }}$. This value is small enough to allow lubrication forces to be effective without leading to its divergence at contact.

\subsection{Drum flow}

The rotating drum is a cylinder of length $L$ and diameter $d_{c}$ constructed geometrically by the juxtaposition of polyhedral rigid elements. Its both ends are closed by two planes and it can rotate around its axis at given angular speed $\omega$. In all simulations analyzed below, the drum is horizontal, implying that the gravity is perpendicular to the drum axis.

The drum is filled by allowing 5000 dry particles to fall into it under their own weights until a stabilized granular bed is obtained. The number of particles was limited to 5000 in order to simulate a large number of drum rotations and many runs with different values of material parameters in a reasonable computation time. In all simulations, the filling level was $s_{f}=\frac{2 H}{d_{c}} \simeq 0.56$, where $H$ is the filling height of the granular material inside the drum. We considered three different size classes in a range $\left[d_{\min }, d_{\max }\right]$ with a size ratio $\alpha=d_{\max } / d_{\min }$. All size classes have the same particle volume so that the size distribution is uniform in terms of particle volume fractions. This corresponds to a small number of large particles and a large number of small particles. This distribution generally leads to a large packing fraction since small particles optimally fill the pore space between the large particles [78, 79]. The mean particle diameter $\langle d\rangle$ can also be defined as a function of $\alpha$ by

$$
\langle d\rangle=d_{\min } \frac{2 \alpha}{1+\alpha}=d_{\max } \frac{2}{1+\alpha} .
$$

Figure 3(a) shows an example of the initial state in a system with $\alpha=5$ and $\langle d\rangle=16 \mu \mathrm{m}$. Figure 3(b) displays the granular flow after 50 rotations. At the beginning, all particles are at rest inside the drum. With drum rotation, a stable flow configuration is reached after nearly four rotations. In particular, in this steady regime the inclination of the free surface remains constant.

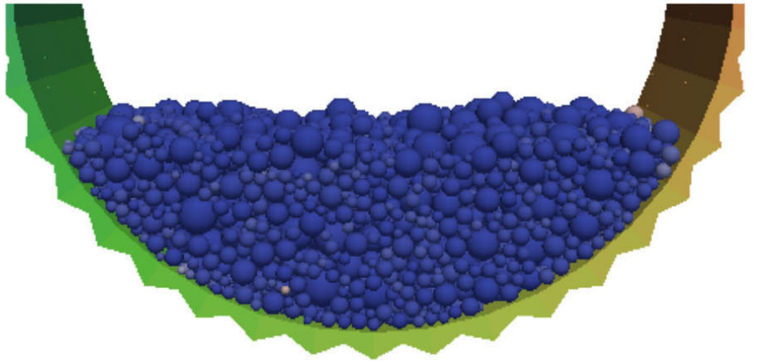

(a)

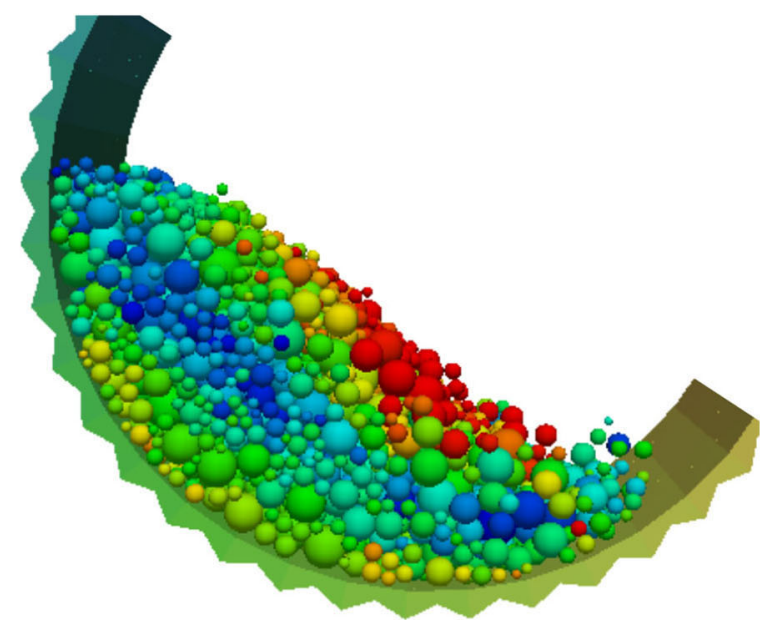

(b)

Fig. 3. Snapshots of the initial state (a) and stable flow (b) in the drum for $\alpha=5$. The colors show the magnitudes of particle velocities varying from red (fast particles at the free surface) to blue (slowest particles in the middle of the drum).

A number $N_{w}$ of particles distributed randomly inside the drum are wetted by attributing them a given amount of liquid. These wet particles may collide and nucleate into small granules during the steady flow of the particles. However, because of the relatively low number of particles, it is numerically more efficient to start the simulations with a single granule introduced initially in the center of the granular bed. The agglomeration process can then be analyzed by following the particles captured by (accretion) or removed from (erosion) the granule. Obviously, the coalescence of granules can be investigated only by simulations with a much larger number of granules. In this paper, we focus on the first option with agglomeration around a single wet granule. To define the initial wet granule, we place a spherical probe in the centre of the granular bed with a radius such that exactly $N_{g 0}=100$ particles are inside the probe. All these particles are considered to be wet. In addition, we randomly select $N_{w}-N_{g 0}=200$ free particles throughout the sample and consider them to be wet. Figure 4 displays the initial state of the granular bed together with the initial granule and free wet particles. The capillary and viscous forces are activated for all wet particles.

The liquid content of wet particles $w=V_{\ell} / V_{g}$, where $V_{\ell}$ is the amount of the liquid and $V_{g}$ is the particle 


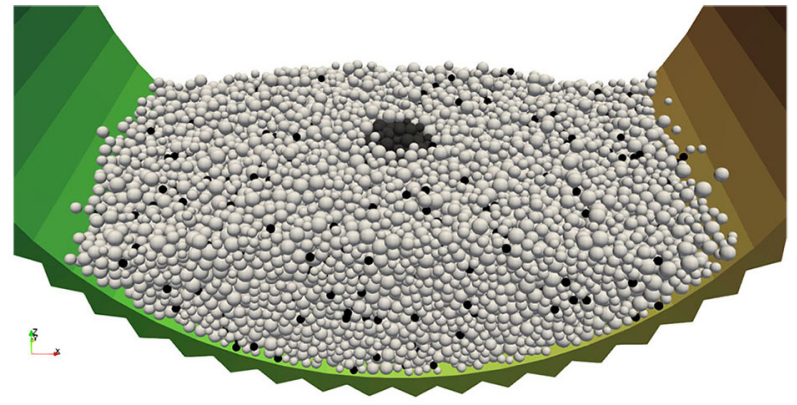

(a)

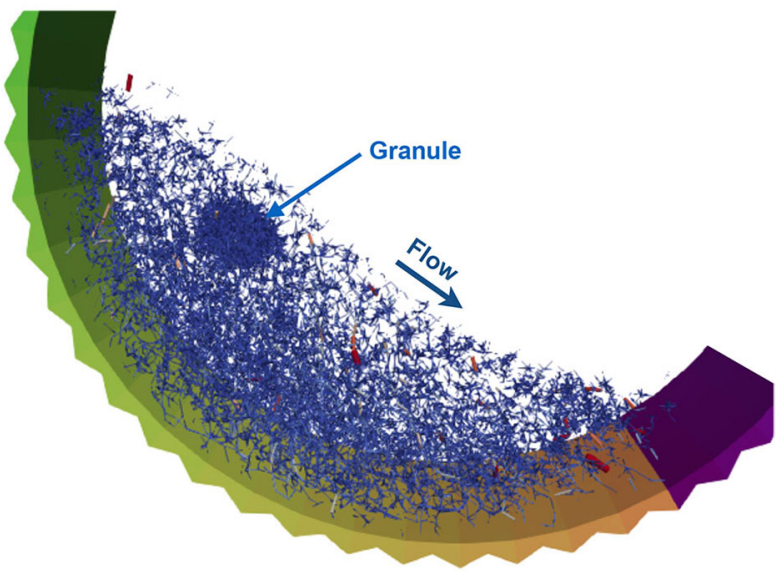

(b)

Fig. 4. (a) Snapshot of the granular bed showing the distribution of dry particles (in white) and wet particles (in black) both those inside the initially defined granule in the center of the bed and those randomly distributed throughout the bed; (b) Force chains during agglomeration: line thickness is proportional to normal force between neighboring particles with compressive forces in blue and tensile forces in red.

volume, is assumed to have the same value for all wet particles. In all our simulations, we set $w=0.09$, which is sufficient to create wet granules in a horizontal rotating drum [7]. When two wet particles meet, the volume of the liquid bridge is $V_{\ell}=w V_{g}$. We also assume that there is no excess liquid so that a wet particle cannot form a liquid bridge with a dry particle.

We performed a large number of simulations with different values of $d_{\min }$ and $d_{\max }$ in the range $[10,1500] \mu \mathrm{m}$, different values of friction coefficient $\mu$ in the range $[0.1,0.9]$ and four values of liquid viscosity $\eta$. For each set of values, several independent granular beds were generated. All values of the material parameters used in our simulations are listed in table 1 . The choice of most parameter values is guided either by numerical efficiency or by reference to the agglomeration process of iron ores in a rotating drum, e.g. for the density of particles, contact angle and filling level. The contact stiffness is chosen such that the elastic deflection at contact points under the weight of the particles in the center of the drum is below 0.01. This ensures the approximation of nearly rigid particles. The damping parameter is chosen for a restitution coefficient of 0.1. However, for data analysis we rely on dimensionless
Table 1. Simulation parameters.

\begin{tabular}{lll}
\hline Parameter & Symbol & Value and unit \\
\hline Particle diameters & $d$ & {$[10,1500] \mu \mathrm{m}$} \\
Density of particles & $\rho$ & $3500 \mathrm{~kg} \cdot \mathrm{m}^{-3}$ \\
Size ratios & $\alpha$ & {$[1,5]$} \\
Number of particles & $N_{p}$ & 5000 \\
Filling level & $s_{f}$ & 0.56 \\
Friction coefficient & $\mu$ & {$[0.1,0.9]$} \\
Normal stiffness & $k_{n}$ & $100 \mathrm{~N} / \mathrm{m}$ \\
Tangential stiffness & $k_{t}$ & $80 \mathrm{~N} / \mathrm{m}$ \\
Normal damping & $\gamma_{n}$ & $5 \cdot 10^{-5} \mathrm{Ns} / \mathrm{m}$ \\
Tangential damping & $\gamma_{t}$ & $5 \cdot 10^{-5} \mathrm{Ns} / \mathrm{m}$ \\
Surface tension & $\gamma_{s}$ & $0.021 \mathrm{~N} / \mathrm{m}$ \\
Contact angle & $\theta$ & $4.0 \mathrm{degree}$ \\
Liquid viscosity & $\eta$ & {$[10 ; 20 ; 40 ; 60] \mathrm{mPa} \cdot \mathrm{s}$} \\
Time step & $\delta t$ & $10^{-7} \mathrm{~s}$ \\
\hline
\end{tabular}

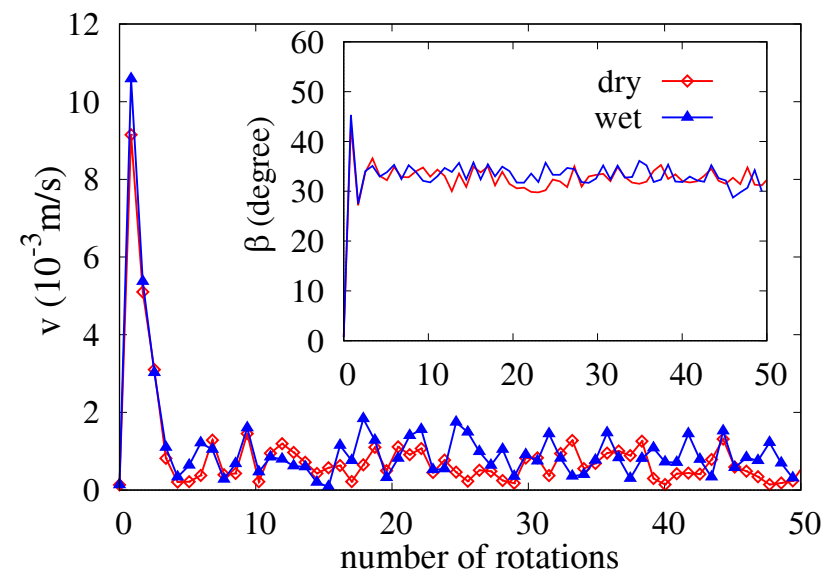

Fig. 5. The mean velocity $v$ of drum flow for dry particles and in the presence of wet particles with $F r=0.5$ as a function of the number of rotations of the drum. The inset shows the evolution of the free surface inclination angle $\beta$ as a function of the number of rotations.

parameters. For rotating drum, a relevant dimensionless parameter is the Froude number $\operatorname{Fr}[44,45,80]$ :

$$
F r=\frac{\omega^{2} d_{c}}{2 g} .
$$

The flow regime mainly depends on the value of the Froude number. For dry particles, the value $F r=0.5$ leads to a flow regime intermediate between rolling and cascading regimes $[26,66]$. Since these regimes have been established for dry particles, we investigated the effect of the wet particles by comparing the velocity profiles for dry particles, on the one hand, and in the presence of wet particles, on the other hand, for $F r=0.5$. We observe practically the same velocity profiles in both cases. Figure 5 shows the mean particle velocity $v$ and free surface inclination $\beta$ for the two simulations with $\alpha=5$. We see that both $v$ and 


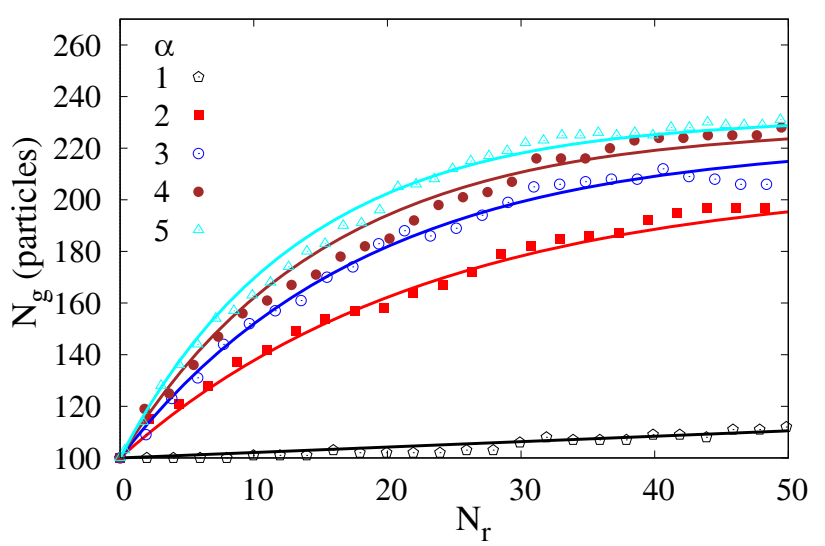

Fig. 6. Evolution of the granule size $N_{g}$ (in number of particles) for different values of size ratio $\alpha$ and $d_{\min }=10 \mu \mathrm{m}$. The solid lines are exponential fits given by eq. (16).

$\beta$ have the same value in both flows. The only difference is that there are more fluctuations in the presence of free wet particles that can be attributed to the higher inhomogeneity of the flow in this case.

\section{Growth, accretion and erosion}

In this section, we are interested in the evolution of the granule size, in terms of the total number of particles $N_{g}$ embodied in the granule, as well as the relative contributions of the accretion and erosion events. The cumulative accretion is the number $N_{g}^{+}$of free wet particles captured by the granule whereas the cumulative erosion $N_{g}^{-}$is the number of wet particles leaving the granule. The rates of these events depend on the relative importance of force chains and cohesive stresses acting on the granule. The values of the process parameters affect the rates so that the granule may grow at different rates. When the rate becomes negative, the granule initially inserted into the granular bed will disappear by excess erosion. Unless explicitly stated, the liquid properties are those of water $\left(\eta=1 \mathrm{mPa} \cdot \mathrm{s}\right.$ and $\left.\gamma_{s}=0.072 \mathrm{~N} / \mathrm{m}\right)$. From the parametric study, we will determine the phase diagram of granule growth for size polydispersity vs. mean particle size.

Figure 6 shows the evolution of the granule size as a function of the number of drum rotations $N_{r}=\omega t / 2 \pi$, where $t$ is the agglomeration time, for different values of the size ratio $\alpha, F r=0.5$ and $\mu=0.5$. In these simulations, $d_{\min }$ is kept constant and equal to $10 \mu \mathrm{m}$. This means that $\alpha$ is increased here by increasing $d_{\max }=$ $\alpha d_{\text {min }}$. We see that the granule grows with $N_{r}$ at a rate that increases with $\alpha$ and levels off as the number of wet particles is exhausted. The increase of agglomeration rate with increased size polydispersity is more spectacular when compared to the mono-disperse case $(\alpha=1)$ in which the granule growth is negligibly small after 50 rotations. As we are interested in this paper in the evolution of captured and eroded particles, the granule size $N_{g}$ is expressed in terms of the number of wet particles in the

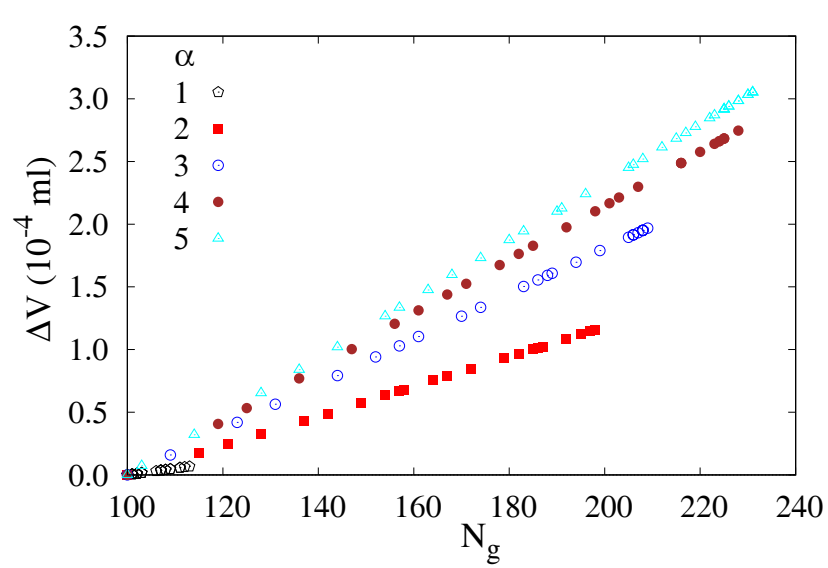

Fig. 7. Variation $\Delta V$ of the volume of particles inside the granule as a function of their number $N_{g}$ for different values of size ratio $\alpha$ and $d_{\min }=10 \mu \mathrm{m}$

granule. Even for broad size polydispersity, we find that the total volume of wet particles belonging to the granule is a linear function of their number, as shown in fig. 7, up to a factor that depends on the size polydispersity. Hence, the trends of $N_{g}$ investigated below are similar to those of the total volume of the granule or the total volume of particles in the granule.

The cumulative accreted and eroded particles are plotted in fig. 8 as a function of $N_{r}$ only for polydisperse samples in which we have a significant number of particles captured and eroded. The accretion grows at a negative rate whereas erosion is a linear function. We will see below with other values of material parameters that the erosion can grow in a non-linear way and faster than accretion so that the linear evolution observed in fig. 8 may be considered as a first-order effect in the limit of low erosion rates. Figure 8 also shows that both accretion and erosion increase with $\alpha$ to an extent that is higher for accretion than for erosion.

The increase of the number of accreted particles $N_{g}^{+}$at a negative rate is a consequence of the decreasing number of available free wet particles in the granular bed while they are captured by the granule. The number of free wet particles is given by $N_{w}-N_{g}$, where $N_{w}$ is the total number of wet particles including those belonging to the granule. This is equivalent to the decrease of the available liquid for agglomeration as the granules grow. Hence, in the steady flow, we may assume that the variation $\Delta N_{g}^{+}$of the captured particles is proportional to the current number $N_{w}-N_{g}$ of wet particles and to the elapsed time $\Delta t$ or angular rotation $\Delta N_{r}=\omega \Delta t$

$$
\Delta N_{g}^{+}=k^{+} \frac{N_{w}-N_{g}}{N_{w}} \Delta N_{r}
$$

where $k^{+}$is the relative accretion rate. As to the number $N_{g}^{-}$of eroded particles, we assume that its rate $k^{-}$is constant

$$
\Delta N_{g}^{-}=k^{-} \Delta N_{r}
$$

These equations are consistent with our numerical data shown in fig. 8 although we expected the number of eroded 


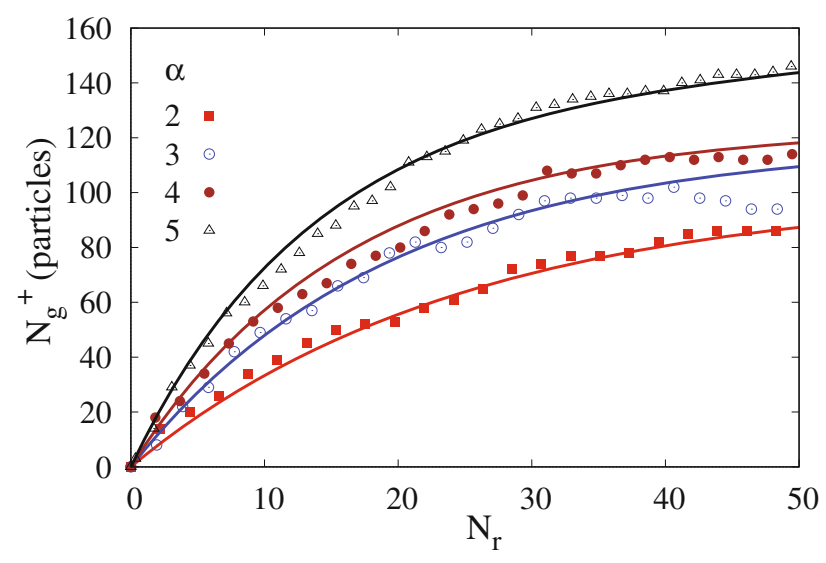

(a)

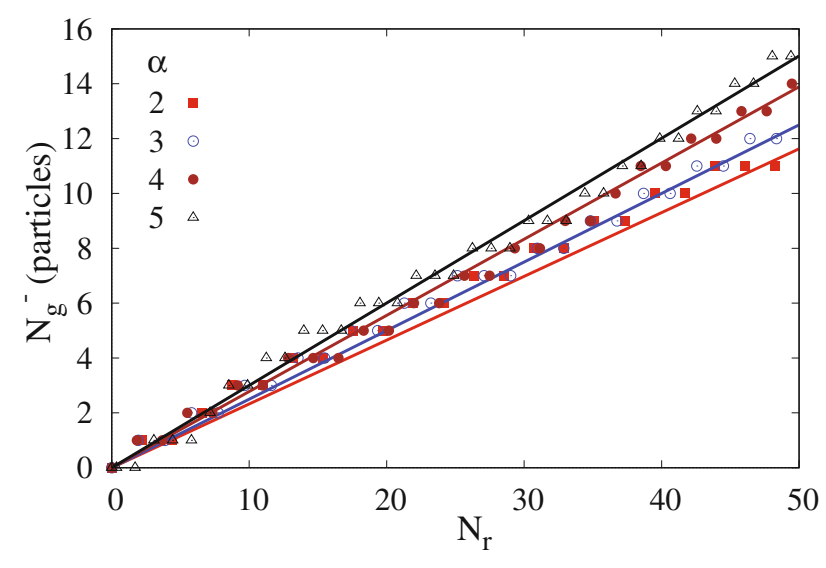

(b)

Fig. 8. Cumulative accretion (a) and cumulative erosion (b) of particles for different values of size ratio $\alpha$. The lines are fitting forms given by eqs. (17) and (18).

particles to be proportional to the number of particles at the surface of the granule. This may reflect the fact that the average curvature of the granule surface diminishes as its size increases so that the particles lying at the surface of the granule are more strongly attached to the granule and less subjected to the eroding action of granular flow. This effect may counter-balance the increase of the granule surface area. However, for much larger granules in number of primary particles this effect may disappear.

With the above assumptions, the rate equation for the granule size is simply $\Delta N_{g}=\Delta N_{g}^{+}-\Delta N_{g}^{-}=$ $\left(-k^{+} N_{g} / N_{w}+k^{+}-k^{-}\right) \Delta N_{r}$, which leads to a simple differential equation,

$$
\frac{\mathrm{d} N_{g}}{\mathrm{~d} N_{r}}=k^{+}\left(1-N_{g} / N_{w}\right)-k^{-}
$$

with the following solution:

$N_{g}\left(N_{r}\right)=N_{g 0}+\left\{N_{w}\left(1-k^{-} / k^{+}\right)-N_{g 0}\right\}\left(1-e^{\frac{-k^{+}}{N_{w}}} N_{r}\right)$.

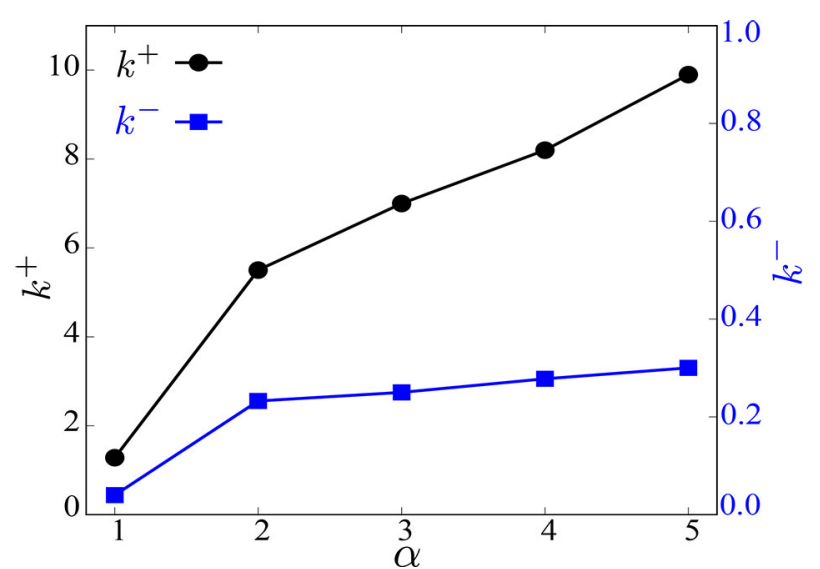

Fig. 9. Fitted values of erosion rate $k^{-}$and accretion rate $k^{+}$ as a function of polydispersity parameter $\alpha$.

This model predicts an exponential growth and an asymptotic granule size $N_{g}(t \rightarrow \infty)=N_{w}\left(1-k^{-} / k^{+}\right)$, as observed in our simulations. A similar growth and level-off is observed in experiments with a finite amount of available liquid although the experimental conditions and the agglomeration method are not the same as in our simulations $[42,81]$. The steady agglomeration state corresponds to the condition $\Delta N_{g}^{+}=\Delta N_{g}^{-}$. For $k^{-} \ll k^{+}$, the final granule embodies nearly all wet particles: $N_{g} \simeq N_{w}$. On the other hand, the granule disappears if $k^{-}>k^{+}$.

The evolution of $N_{g}^{+}$and $N_{g}^{-}$as a function of drum rotation can be obtained from eqs. (16), (13) and (14). We get

$$
N_{g}^{-}=k^{-} N_{r}
$$

and

$$
N_{g}^{+}=\left\{N_{w}\left(1-k^{-} / k^{+}\right)-N_{g 0}\right\}\left(1-e^{\frac{-k^{+}}{N_{w}}} N_{r}\right)+k^{-} N_{r} .
$$

In view of the present model, the influence of particle size ratio $\alpha$ observed in figs. 6 and 8 can be interpreted in terms of the accretion and erosion rates. Figure 9 shows the fitted values of $k^{-}$and $k^{+}$as a function of $\alpha$. The increase of accretion rate $k^{+}$with $\alpha$ is rather counter-intuitive since the cohesive strength is inversely proportional to the mean particle size, which increases here with $\alpha$. This means that the higher polydispersity, allowing for a better filling of the pore space and thus higher density of the granule, overcompensates the decrease of the cohesive stress. But the latter explains the increase of the erosion rate $k^{-}$, which is quite small compared to $k^{+}$.

\section{Effects of material parameters}

We now consider the effect of the mean particle size $\langle d\rangle$, which directly controls the cohesive stress of wet particles. Figure 10 displays the cumulative number of particles for both accretion and erosion in the case $\alpha=5, \mu=0.5$. Note that, in these simulations, the higher values of $\langle d\rangle$ 


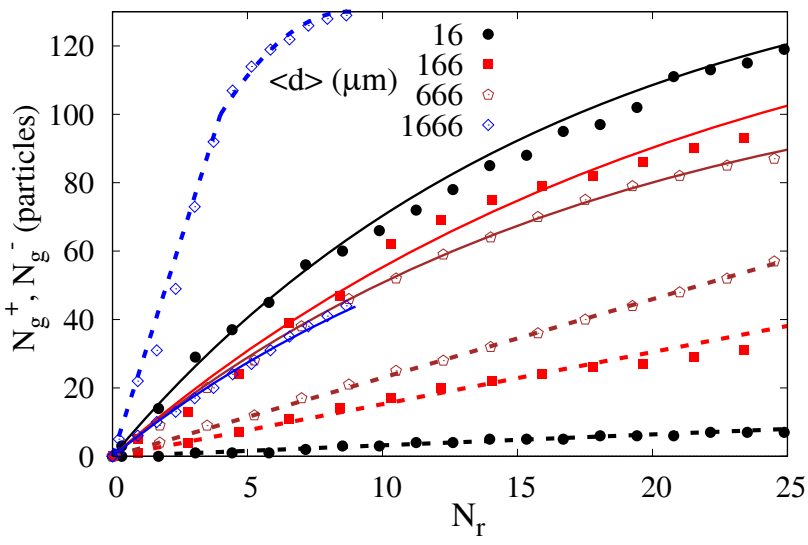

Fig. 10. Evolution of the cumulative number of wet particles for accretion (solid lines) and erosion (dashed lines) for four different values of the mean particle diameter $\langle d\rangle$ and size ratio $\alpha=5$, as a function of the number of drum rotations.

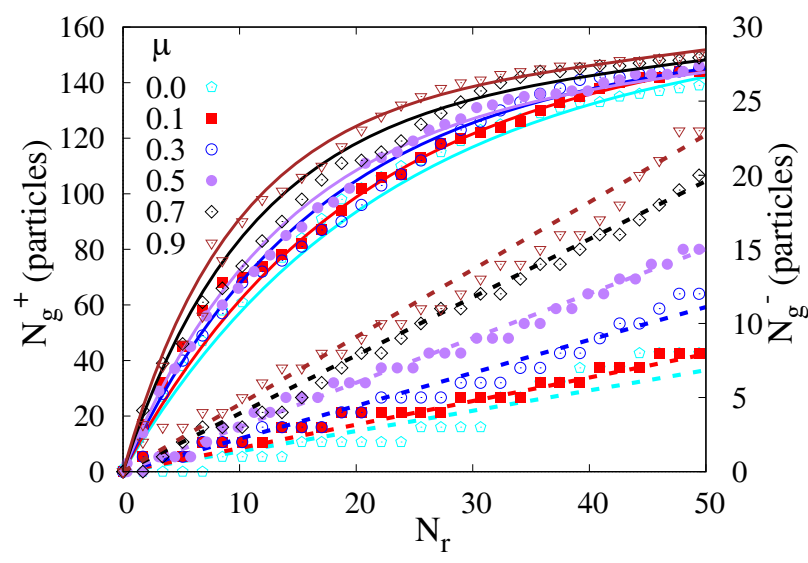

Fig. 11. Cumulative accretion (solid lines) and erosion (dashed lines) of wet particles for five different values of the friction coefficient $\mu$, size ratio $\alpha=5$ and $d_{\min }=10 \mu \mathrm{m}$, as a function of the number of drum rotations. The lines are fitting forms given by eqs. (17) and (18).

imply higher values of both $d_{\min }$ and $d_{\max }$. But, according to eq. (11), $d_{\max }$ increases faster than $d_{\text {min }}$. Figure 10 shows that accretion $N_{g}^{+}$increases as an exponential function of the number of drum rotations whereas erosion $N_{g}^{-}$ is quasi-linear. As expected, since the cohesive stress declines, accretion decreases and erosion increases with increasing $\langle d\rangle$. For $\langle d\rangle=1666 \mu \mathrm{m}$, erosion is high enough to cancel the effect of accretion, and thus the granule disappears after 8 rotations. Here, the cumulative erosion does not grow linearly with time and for this reason the erosion rate is not constant. For the linear part of erosion, we have $k^{-} \simeq 25$ whereas $k^{+} \simeq 6$. Clearly, $k^{-}$is strongly dependent on the cohesive stress of the granule, which declines in inverse proportion to the wet particle mean size. The cohesive stress of wet particles affects, albeit to a lesser extent, the accretion rate $k^{+}$.

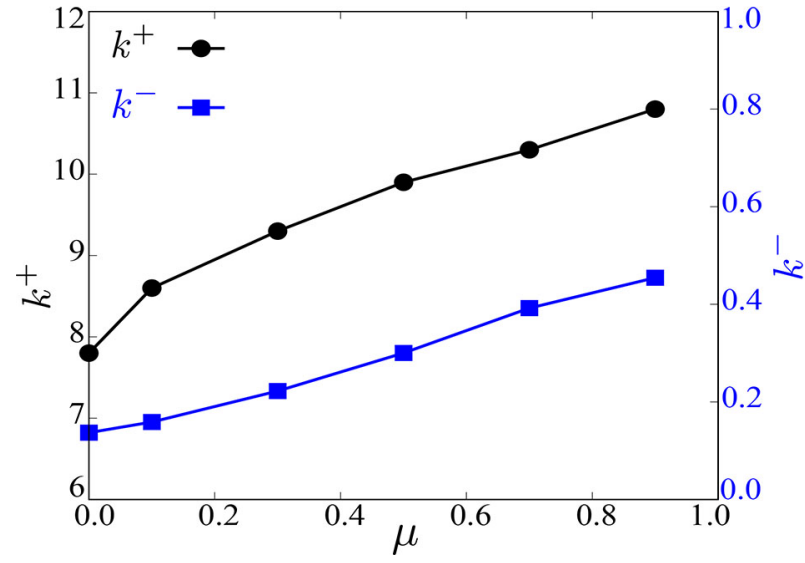

Fig. 12. Fitted values of erosion rate $k^{-}$and accretion rate $k^{+}$as a function of friction coefficient $\mu$ for size ratio $\alpha=5$.

The friction coefficient $\mu$ between particles is a major parameter for granular flows. Its joint effect with cohesive forces can thus influence the agglomeration process. Figure 11 shows cumulative accretion and erosion for an increasing value of $\mu$ and exponential fits. Figure 12 displays $k^{-}$and $k^{+}$as a function of $\mu$. We observe a slight increase of both rates. The values of $N_{g}^{+}$and $N_{g}^{-}$after 50 rotations show that accretion increases slightly with $\mu$, which may be understood as a consequence of enhanced capturing of free wet particles when they touch the granule. However, $N_{g}^{-}$increases to a larger extent with $\mu$ so that the granule growth is slower at larger values of $\mu$. As erosion is a consequence of interactions between dry particles and boundary wet particles of the granule, the increase of erosion with $\mu$ may be understood as an increase of shear forces acting on the boundary particles by shear flow of dry particles. This suggests that the agglomeration of rough particles (with higher friction coefficient) is less efficient than smooth particles and it should consume more energy.

This brings us to the effect of liquid viscosity expressed as a lubrication force between wet particles. We performed several simulations for different values of $\eta$ in the range $[10,60] \mathrm{mPa} \cdot \mathrm{s}$ and with same value of the liquid-vapor surface tension $\gamma_{s}=21 \mathrm{mN} / \mathrm{m}$. Figure 13 shows the evolution of cumulative accretion and erosion for $\alpha=5$. Again, we observe the exponential increase of $N_{g}^{+} v s$. the nearly linear increase of $N_{g}^{-}$with the number of drum rotations. The accretion increases slightly with $\eta$ whereas the erosion declines. The decrease of $N_{g}^{-}$is exactly the opposite effect of friction coefficient in fig. 11 which causes an increase of $N_{g}^{-}$. This means that lubrication forces tend to reduce the shearing effect of the flow on the granule, leading to smaller erosion. On the other hand, the increase of accretion can be attributed to viscous dissipation that can enhance the capture of free wet particles by the granule. Figure 14 shows $k^{-}$and $k^{+}$as a function of $\eta$. The variations of the rates with $\eta$ are small but their opposite effects tend to enhance granule growth. It is also interesting to note that the linear decrease of $k^{-}$was also observed in experiments [66]. 


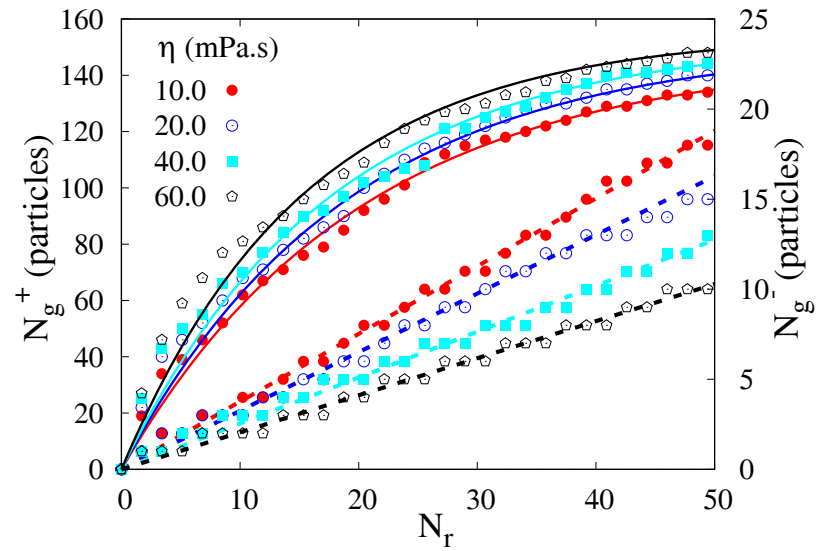

Fig. 13. Cumulative accretion (solid lines) and erosion (dashed lines) for four different values of the liquid viscosity $\eta$ for size ratio $\alpha=5$ and $d_{\text {min }}=10 \mu \mathrm{m}$ as a function of the number of drum rotations. The solid and dashed lines are exponential and linear fits to the data points, respectively.

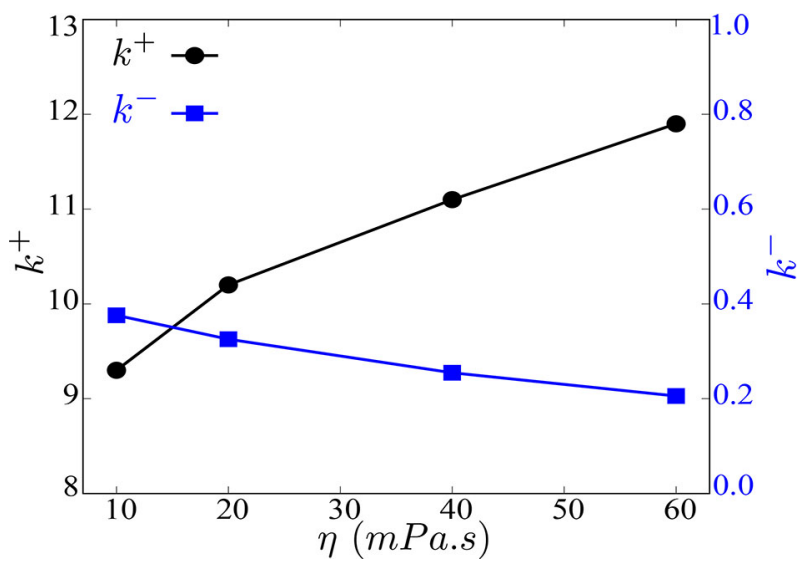

Fig. 14. Erosion rate $k^{-}$and accretion rate $k^{+}$as a function of liquid viscosity $\eta$ for size ratio $\alpha=5$ and $d_{\text {min }}=10 \mu \mathrm{m}$.

\section{Phase diagram}

In the simulations reported in this paper, we have a single granule of size $N_{g 0}$ that can increase or decrease in size depending on the values of various material parameters. In the last section, we analyzed the influence of several parameters on the accretion and erosion rates. The most crucial issue, however, in this single-granule problem is the ranges of the values of those parameters for which the granule will survive and grow, i.e. the phase diagram of agglomeration. We consider here only the phase diagram in the parameter space of the mean particle size $\langle d\rangle v s$. size ratio $\alpha$. In these simulations, we set $\eta=1, \gamma_{s}=72 \mathrm{mN} / \mathrm{m}$ and $\mu=0.5$.

Figure 15 displays all the simulated points as a grid in the parameter space $[\langle d\rangle, \alpha]$ and an approximate frontier between the range of values for which the granule survives, i.e. agglomeration is possible, and the values for which no granule can grow. We see that for $\langle d\rangle>1200 \mu \mathrm{m}$ no agglomeration occurs even for large values of $\alpha$ (larger polydispersity). For smaller values of $\langle d\rangle$, the range of the

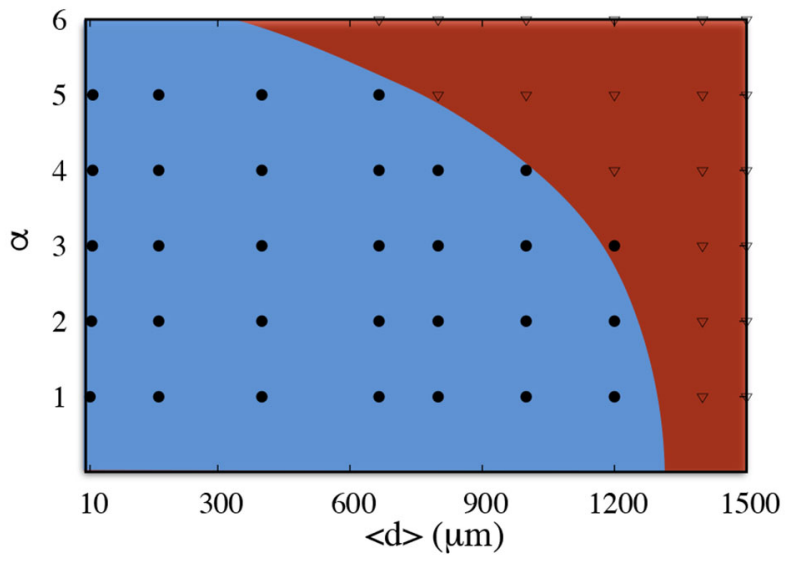

Fig. 15. Phase diagram of granule growth in the parametric space of $\langle d\rangle$ vs. $\alpha$ for $\eta=1 \mathrm{mPa} \cdot \mathrm{s}$ and $\mu=0.5$. The granule grows only in the light blue region and disappears otherwise.

values of $\alpha$ for agglomeration increases. Note that the limit on the value of $\alpha$ is related to the fact that for given $\langle d\rangle$, the increase of $\alpha$ requires the increase of $d_{\max }$ and decrease of $d_{\min }$. The increase of $d_{\max }$ compensates to some extent the effect of decreasing $d_{\min }$ on the cohesive stress.

\section{Conclusions}

In this paper, we used a 3D DEM algorithm with a capillary cohesion law enhanced by the viscous effect of the binding liquid in order to investigate the agglomeration of wet particles in dense granular flows inside a rotating drum. The system was numerically prepared by pouring solid particles into a drum composed of polyhedral elements. We considered in detail the evolution of a single granule acting for a broad range of parameter values. The liquid was assumed to be transported by the particles and its amount was defined by the number of initial wet particles in the granule and the number of free wet particles randomly distributed inside the granular bed.

We showed that the granule grows almost exponentially with time (or the number of drum rotations) as a result of a gradual capture of free wet particles by the granule. The accretion of free wet particles is nearly always an exponential function of time whereas the number of eroded particles grows linearly with time. A simple model based on constant accretion and erosion rates was introduced, predicting the observed exponential increase of granule size that levels off to a constant value at long times. We investigated the effects of size ratio, mean particle size, friction coefficient and liquid viscosity on accretion and erosion of particles. Both accretion and erosion increase when the size ratio or friction coefficient are increased. Accretion declines whereas erosion increases when the mean particle size is increased. Accretion increases whereas erosion declines when liquid viscosity is increased. We determined the phase diagram of agglomeration by varying systematically the size ratio and mean particle size. This will be extended to other parameters in the future. 
Our results are based on a simple system with a relatively small number of spherical particles. We considered the evolution of a single granule and this allowed us to perform long-time simulations for a broad range of the values of material parameters. Clearly, these simulations can be extended to obtain the combined effects of parameters in phase-space diagrams. The effects of process parameters such as filling rate, rotation speed and wetting procedure can be studied, too. Further data analysis is also necessary in order to investigate the granule consolidation and nucleation from free wet particles when no granule is initially introduced. Despite its higher computational cost, a larger number of particles may allow for the simulation of multi-granule systems and coalescence phenomena.

We gratefully acknowledge financial support by the Ministry of Education and Training in Vietnam and Campus France. This work was initiated in collaboration with ArcelorMittal.

\section{Author contribution statement}

All the authors were involved in the preparation of the manuscript. All the authors have read and approved the final manuscript.

\section{References}

1. S.M. Iveson, J.D. Litster, K. Hapgood, B.J. Ennis, Powder Technol. 117, 3 (2001).

2. S.H. Chien, G. Carmona, L.I. Prochnow, E.R. Austin, J. Environ. Qual. 32, 1911 (2003).

3. A. Barkouti, E. Rondet, M. Delalonde, T. Ruiz, J. Food Eng. 111, 234 (2012).

4. E. Rondet, M. Delalonde, T. Ruiz, J.P. Desfoursb, Chem. Eng. J. 164, 376 (2010).

5. A. Nosrati, J. Addai-Mensah, D.J. Robinson, Hydrometallurgy 125-126, 90 (2012).

6. K.V. Sastry, P. Dontula, C. Hosten, Powder Technol. 130 , 231 (2003).

7. R. Aguado, S. Roudier, L. Delagado (Editors), Best available techniques (BAT) reference document for iron and steel production (Joint Research Centre of the European Commission, Publications Office of the European Union, Luxembourg, 2013).

8. G.M. Walker, Handb. Powder Technol. 11, 219 (2007) (Granulation).

9. K. Saleh, P. Guigon, Handb. Powder Technol. 11, 323 (2007) (Granulation).

10. P. Suresh, I. Sreedhar, R. Vaidhiswaran, A. Venugopal, Chem. Eng. J. 328, 785815 (2017).

11. S. Herminghaus, Adv. Phys. 54, 221 (2005).

12. J. Litster, B. Ennis, The Science and Engineering of Granulation Processes, Vol. 15 (Springer, Netherlands, 2014).
13. C. Liao, S. Hsiau, S. Wen, Adv. Powder Technol. 27, 1265 (2016).

14. S. Iveson, J. Litster, AIChE J. 44, 1510 (1998).

15. X. Xiao, Y. Tan, H. Zhang, R. Deng, S. Jiang, Powder Technol. 314, 182 (2017).

16. B. Alchikh-Sulaiman, M. Alian, F. Ein-Mozaffari, A. Lohi, S.R. Upreti, Particuology 25, 133 (2016).

17. A. Bouwman, M. Henstra, D. Westerman, J. Chung, Z Zhang, A. Ingram, J. Seville, H. Frijlink, Int. J. Pharm. 290, 129 (2005).

18. J. Degrève, J. Baeyens, M.V. de Velden, S.D. Laet, Powder Technol. 163, 188 (2006).

19. J.D. Osborne, R.P. Sochon, J.J. Cartwright, D.G. Doughty, M.J. Hounslow, A.D. Salman, Chem. Eng. Res. Des. 89, 553 (2011).

20. S.K. Pawar, F. Henrikson, G. Finotello, J.T. Padding, N.G. Deen, A. Jongsma, F. Innings, J.H. Kuipers, Powder Technol. 300, 157 (2016).

21. R. Pashminehazar, A. Kharaghani, E. Tsotsas, Powder Technol. 300, 46 (2016).

22. B.J. Ennis, G. Tardos, R. Pfeffer, Powder Technol. 65, 257 (1991).

23. F. Štěpánek, P. Rajniak, C. Mancinelli, R. Chern, R. Ramachandran, Powder Technol. 189, 376 (2009).

24. A. Hassanpour, M. Pasha, L. Susana, N. Rahmanian, A.C. Santomaso, M. Ghadiri, Powder Technol. 238, 50 (2013).

25. N. Rahmanian, A. Naji, M. Ghadiri, Chem. Eng. Res. Des. 89, 512 (2011).

26. B.J. Ennis, Powder Technol. 88, 203 (1996).

27. M. Butensky, D. Hyman, Ind. Eng. Chem. Fundam. 10, $212(1971)$

28. K.E. Ileleji, Y. Li, R.K. Ambrose, P.H. Doane, Powder Technol. 300, 126 (2016).

29. R. Ramachandran, J.M.H. Poon, C.F. Sanders, T. Glaser, C.D. Immanuel, F.J. Doyle, J.D. Litster, F. Stepanek, F.Y. Wang, I.T. Cameron, Powder Technol. 188, 89 (2008).

30. J.P. Pan, T.J. Wang, J.J. Yao, Y. Jin, Powder Technol. 162, 50 (2006).

31. R. Spurling, J. Davidson, D. Scott, Chem. Eng. Res. Des. 79, 51 (2001).

32. F. Wang, I. Cameron, Powder Technol. 179, 2 (2007).

33. J.M.N.T. Gray, J. Fluid Mech. 441, 1 (2001).

34. C. Thornton, Z. Ning, Powder Technol. 99, 154 (1998).

35. L.X. Liu, J.D. Litster, S.M. Iveson, B.J. Ennis, AIChE J. 46, 529 (2000).

36. S. Iveson, J. Beathe, N. Page, Powder Technol. 127, 149 (2002).

37. M. Ghadiri, A.D. Salman, M. Hounslow, A. Hassanpour, D.W. York, Chem. Eng. Res. Des. 89, 499 (2011)

38. J. Rojek, S. Nosewicz, M. Maździarz, P. Kowalczyk, K. Wawrzyk, D. Lumelskyj, Procedia Eng. 177, 263 (2017).

39. GDR-MiDi, Eur. Phys. J. E 14, 341 (2004).

40. K. Kamrin, D.L. Henann, Soft Matter 11, 179 (2015).

41. L. Amarsid, J.Y. Delenne, P. Mutabaruka, Y. Monerie, F. Perales, F. Radjai, Phys. Rev. E 96, 012901 (2017).

42. M.A. Behjani, N. Rahmanian, N.F. bt Abdul Ghani, A. Hassanpour, Adv. Powder Technol. 28, 2456 (2017).

43. I. Govender, Miner. Eng. 92, 168 (2016).

44. R. Yang, A. Yu, L. McElroy, J. Bao, Powder Technol. 188, $170(2008)$

45. J. Mellmann, Powder Technol. 118, 251 (2001).

46. G. Lian, C. Thornton, M. Adams, J. Colloid Interface Sci. 161, 138 (1993). 
47. M. Scheel, R. Seemann, M. Brinkmann, M.D. Michiel, A. Sheppard, S. Herminghaus, J. Phys.: Condens. Matter 20, 494236 (2008).

48. C. Willett, M. Adans, S. Johnson, J. Seville, Langmuir 16, 9396 (2000).

49. J.Y. Delenne, V. Richefeu, F. Radjai, J. Fluid Mech. 762, R5 (2015).

50. F. Radjai, F. Dubois, Discrete-Element Modeling of Granular Materials (Wiley-Iste, 2011).

51. V. Richefeu, F. Radjai, M.S.E. Youssoufi, Eur. Phys. J. E 21, 359 (2007).

52. I. Talu, G.I. Tardos, M.I. Khan, Powder Technol. 110, 59 (2000).

53. E.L. Chan, K. Washino, G.K. Reynolds, B. Gururajan, M.J. Hounslow, A.D. Salman, Powder Technol. 300, 92 (2016).

54. P. Lau, M. Kind, Powder Technol. 300, 28 (2016).

55. S. Sarkar, B. Chaudhuri, Asian J. Pharm. Sci. 13, 220 (2018).

56. D. Barrasso, A. Tamrakar, R. Ramachandran, Chem. Eng. Sci. 119, 319 (2014).

57. V. Richefeu, M.S. El Youssoufi, E. Azéma, F. Radjai, Powder Technol. 190, 258 (2009).

58. H. Matuttis, S. Luding, H. Herrmann, Powder Technol. 109, 278 (2000).

59. C. Thornton, Géotechnique 50, 43 (2000).

60. F. Radjai, I. Preechawuttipong, R. Peyroux, Cohesive granular texture, in Continuous and Discontinuous Modelling of Cohesive Frictional Materials, edited by P. Vermeer, S. Diebels, W. Ehlers, H. Herrmann, S. Luding, E. Ramm (Springer Verlag, Berlin, 2001) pp. 148-159.

61. H.J. Herrmann, S. Luding, Contin. Mech. Thermodyn. 10, 189 (1998).

62. D. Shi, J. McCarthy, Powder Technol. 184, 64 (2008).

63. B. Mohan, C. Kloss, J. Khinast, S. Radl, Powder Technol. 264, 377 (2014).

64. A. Leonardi, M. Cabrera, F.K. Wittel, R. Kaitna, M. Mendoza, W. Wu, H.J. Herrmann, Phys. Rev. E 92, 052204 (2015).
65. K. Melnikov, R. Mani, F.K. Wittel, M. Thielmann, H.J. Herrmann, Phys. Rev. E 92, 022206 (2015).

66. G. Lefebvre, P. Jop, Phys. Rev. E 8, 032205 (2013).

67. J. Duran, A. Reisinger, P. de Gennes, Sands, Powders, and Grains: An Introduction to the Physics of Granular Materials, Partially Ordered Systems Series (Springer, New York, 1999)

68. V. Richefeu, M. El Youssoufi, F. Radjai, Phys. Rev. E 73 , 051304 (2006).

69. T-Trung. Vo, P. Mutabaruka, J.-Y. Delenne, S. Nezamabadi, F. Radjai, EPJ Web of Conferences 140, 08021 (2017).

70. J. Schäfer, S. Dippel, D.E. Wolf, J. Phys. I France 6, 5 (1996).

71. S. Dippel, G.G. Batrouni, D.E. Wolf, Phys. Rev. E 56, 3645 (1997)

72. S. Luding, Collisions and contacts between two particles, in Physics of Dry Granular Media, NATO Science Series E, Vol. 350, edited by H.J. Herrmann, J.P. Hovi, S. Luding (Springer Netherlands, Dordrecht, 1998) p. 285.

73. V. Richefeu, M.S. El Youssoufi, F. Radjaï, in Theoretical and Numerical Unsaturated Soil Mechanics (Springer, 2007) pp. 83-91.

74. T-Trung. Vo, P. Mutabaruka, S. Nezamabadi, J.Y. Delenne, E. Izard, R. Pellenq, F. Radjai, Mech. Res. Commun. 92, 1 (2018).

75. F. Radjaï, V. Richefeu, Philos. Trans. R. Soc. A 367, 5123 (2009).

76. S. Khamseh, J.N. Roux, F.M.C. Chevoir, Phys. Rev. E 92 , 022201 (2015).

77. J. Happel, H. Brenner, Low Reynolds Number Hydrodynamics (Martinus Nijhoff Publishers, 1983).

78. C. Voivret, F. Radjaï, J.Y. Delenne, M.S.E. Youssoufi, Phys. Rev. E 76, 021301 (2007)

79. C. Voivret, F. Radjai, J.Y. Delenne, M.S.E. Youssoufi, Phys. Rev. Lett. 102, 178001 (2009).

80. P.Y. Liu, R.Y. Yang, A.B. Yu, Granular Matter 15, 427 (2013).

81. S.A. de Koster, K. Pitt, J.D. Litster, R.M. Smith, Powder Technol. 355, 514 (2019). 